Case Report:

\title{
Attenuation of Motor Evoked Potentials Following Prone Positioning
}

\author{
Sotirios Apostolakis ${ }^{1 *}$ (D) Aikaterini Karagianni ${ }^{1}$ (D) Eirini Chiotaki ${ }^{2}$, Konstantinos Vlachos ${ }^{1}$ (D)
}

1. Department of Neurosurgery, KAT General Hospital of Attica, Kifisia, Greece

2. Intraoperative Monitoring Service, GE Scientific, G. Vasios \& Co, loannina, Greece

$\begin{aligned} & \text { Use yourdevice to scan } \\ & \text { and read the article online }\end{aligned}$
Citation: Apostolakis S, Karagianni A, Chiotaki E, Vlachos K. Attenuation of Motor Evoked Potentials Following Prone Posi-
tioning. Iran J Neurosurg. 2020; 6(1):29-34. http://dx.doi.org/10.32598/irjns.6.1.5
dol : http://dx.doi.org/10.32598/irjns.6.1.5

\section{(c) (i) (\$)}

Article info:

Received: 18 Aug 2019

Accepted: 25 Nov 2019

Available Online: 01 Jan 2020

Keywords:

Endoscopic discectomy, Herniated disk, Motor evoked potentials, Prone position, Spine surgery

\section{ABSTRACT}

Background and Importance: Of utmost importance for successful neurosurgical operations is proper patient positioning. This is particularly the case for operations conducted in the prone position, which is associated with several potential complications.

Case Presentation: Here we report the case of a 52-year-old male patient who underwent endoscopic discectomy for L5-S1 herniated disc. Following placement in the prone position, Motor Evoked Potentials (MEP) were significantly asymmetric, with those from the left side being disproportionately low considering his clinical presentation. MEP recordings were ameliorated when the patient was placed in the supine position. Considering the nervous and vascular anatomy of the area, a peripheral insult is highly unlikely to be the cause of the neurophysiological profile of this patient. Instead, compression of the dural sac due to biomechanical alterations of the contents of the spinal canal is the most possible pathogenetic mechanism.

Conclusion: Proper patient positioning is of paramount importance for the success of a neurosurgical operation. Still, even if all precautions are taken, it is possible that insults to neuronal structures can be due to biomechanical alterations of the contents of the spinal canal, secondary to prone positioning.

\section{* Corresponding Author:}




\section{Highlights}

- Of paramount importance for the success of a neurosurgical procedure is proper patient positioning.

- Even following meticulous padding of bony surfaces, potentially devastating complications may arise.

- Biomechanical alterations following prone positioning may exacerbate the compression of the neuronal elements.

\section{Plain Language Summary}

Proper positioning is one of the most crucial determinants of success in neurosurgical operations. The case of a patient with lumbar spine hernia and diminution of motor evoked potentials following placement in the supine position is presented. Biomechanical alterations of the lumbar spine are speculated to be a potential pathogenetic mechanism.

\section{Background and Importance}

roper positioning is one of the most crucial determinants of success in neurosurgical operations. An appropriately positioned patient will provide an unobscured visual field, an easily accessible surgical plane, and effortless and comfortable positioning of the surgical team. However, the role of the positioning of the neurosurgical patient is not limited to the ease of the surgeon but is also critical for the avoidance of post-operative complications. The complex nature of the operation per se frequently undermines its importance. In practice, even the slightest deviation from optimal practice may lead to numerous and often disastrous complications, compromising the outcome of an otherwise successful operation.

One of the most technically and logistically demanding positions is the prone position, which is frequently applied in spinal and posterior fossa procedures [1]. This is because the patient, particularly if obese, is subjected to increased weight loads, increasing the risk for formation of pressure sores or for peripheral nerve injury [2], but also at the same time, limited access to the endotracheal tube, changes in respiratory physiology and tracheal or large vessel compression $[3,4]$ may compromise the administration of proper anesthesia.

Here we report the case of position-related intra-operative diminution of Motor Evoked Potentials (MEPs) in a patient who underwent endoscopic lumbar discectomy in the prone position.

\section{Case Presentation}

A 52-year-old male patient presented at the outpatient department with sciatica and hypoesthesia of the left lower limb. His medical history was unremarkable of any pathology. Pre-operative neurological examination revealed decreased muscle strength during flexion and extension of the thigh bilaterally (with the left side being more affected), as well as decreased dorsiflexion and plantar flexion of the left foot. Magnetic Resonance Imaging (MRI) of the lumbar spine revealed a herniated disc at the L5-S1 level with a free disc fragment in the epidural space compressing the $L 5$ nerve root (Figure 1 A, B) which was scheduled for endoscopic discectomy.

At the operating theatre, after the establishment of a definitive airway, the patient was placed in the typical prone position for lumbar discectomy, with appropriate padding of the bony surfaces and sites of pressure being meticulously taken care of. Baseline Motor Evoked Potentials (MEPs) demonstrated significant asymmetry of the responses between the limbs, with those from the left being disproportionately low, provided the preoperative clinical examination (Figure 2A). This was more prominent for the tibialis anterior, the gastrocnemius and extensor digitalis longus and to a lesser extent for the abductor hallucis and the quadriceps. The patient was returned to the supine position for a wakeup test (Figure 2B). Propofol was discontinued and with time, motor responses gradually improved. The patient was returned to the prone position, when MEPs were deemed satisfactory. Yet, shortly after repositioning, new, though less pronounced, MEP attenuation was observed. At that point, having established once more that every precaution was taken to avoid excessive external compression of vascular or neuronal structures, it 


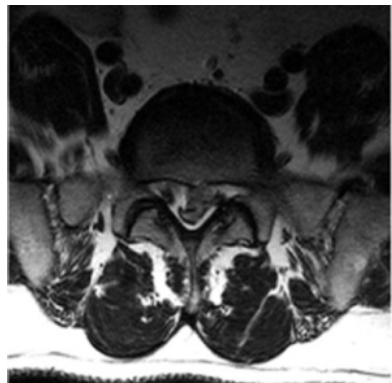

(a)

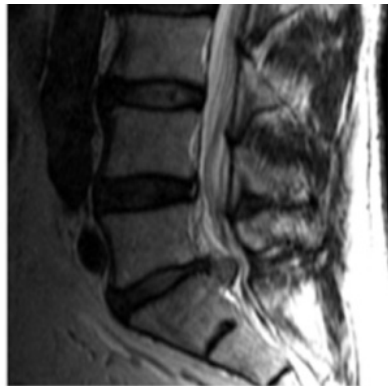

(b)

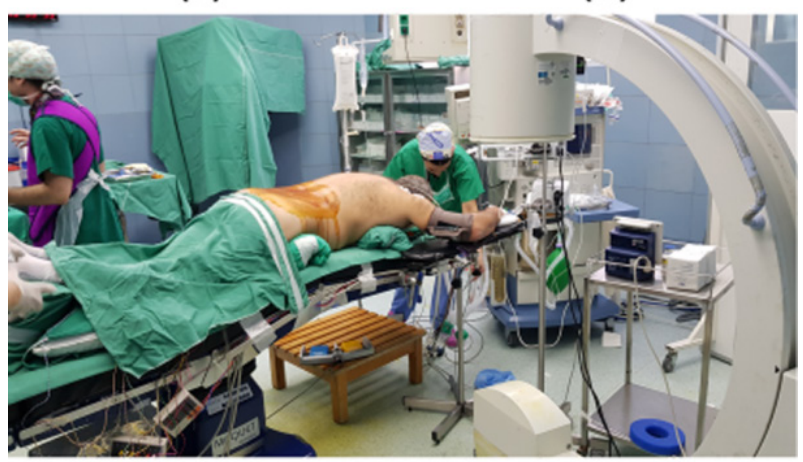

(c)

Figure 1. Pre-operative T2W MRI

A, B: Showed a free disc fragment compressing the dural sac at the level of L5-S1; C. The patient was scheduled for endoscopic discectomy and the standard prone position was applied.

(a)

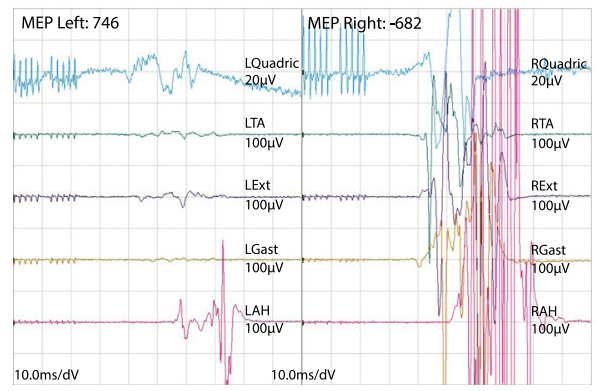

(b)

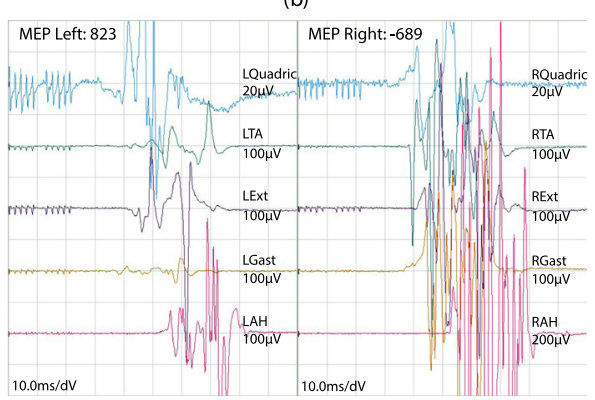

(c)

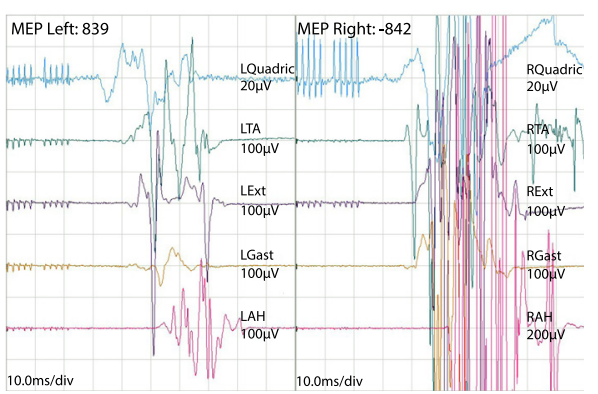

Figure 2. Intra-operative Motor Evoked Potentials (MEP)

A. Initial recordings at prone position, demonstrated attenuation of MEP; B: They were ameliorated following the return to the supine position and discontinuation of propofol; $\mathrm{C}$. The operation was completed without further nuances, and immediate improvement in the MEP was recorded.

L/R denotes left and right side, respectively; Quadric: Quadriceps; TA: Tibialis Anterior; Ext: Extensor Digitorum Longus; Gast: Gastrocnemius; AH: Abductor Hallucis 
was decided to proceed with the operation under continuous neurophysiological monitoring. The operation was completed successfully, without further incidents. Post-operatively, neurophysiological evaluation demonstrated improved MEP of the left lower limb (Figure 2C), while the patient did not demonstrate any additional neurological deficits and was discharged the day following the operation.

\section{Discussion}

Considering the particularities of the case presented, it could be deduced that since MEPs were normalised following the return in the supine position, this diminution is possibly associated with the positioning of the patient on the operating table. Systematic causes of attenuation of MEP include hypotension, hypothermia, increased levels of halogenated inhalational anaesthetics and intravenous sedation, [5]; however, these should lead to bilaterally affected recordings. In particular, the administration of propofol has a dose-dependent effect on motor and somatosensory evoked potentials, decreasing their amplitude bilaterally, whereas no effect has been identified on their latencies [6]. Herein, lack of baseline MEP in the prone position in the absence of propofol, does not allow for an effective evaluation of its effect and thus imposes a great limitation in the present study. Besides, the possibility of hardware malfunction, potential mechanisms could be identified following meticulous investigation of the regional anatomy and of the potential particularities of the case.

As far as the anatomy is concerned, direct insult from compression of vascular structures in the iliac fossa, although rare, has been reported previously [7-11]. Interestingly, some cases were thin adolescents in whom MEPs were progressively attenuated over more than an hour. In turn, direct neuronal compression is primarily an issue when positioning the upper limbs, while it is scarce in peripheral nerves of the lower limbs [12].

Taking into consideration the nature of the pathology under treatment and the particularities of the anatomy of the individual, additional potential pathogenetic mechanisms could be identified. This is particularly the case when significant stenosis of the spinal canal is the reason for the primary complaint of the patient. In such instances, extreme flexion or extension of the spinal cord could be detrimental $[5,13,14]$. Moreover, intraoperative insults to peripheral nerves secondary to stenosis in their course have been documented [15].

The attenuation of MEP in the present case could potentially be attributed to the positioning of the patient since a significant amelioration was observed shortly after the return to the supine position. However, unlike the aforementioned cases, the anatomic location of the muscles affected points towards a pathogenetic mechanism different from vascular or neuronal compression.

In particular, muscles of both the ventral (quadriceps, tibialis anterior, extensor digitorum longus) and dorsal (gastrocnemius) compartments of the leg were affected, including the foot (abductor hallucis), innervated by different peripheral nerves (Table 1) and vascularised from various feeding arteries, in which case only occlusion of the iliac artery could lead to the neurophysiologic picture. This is because branches of the internal iliac artery provide sufficient collateral supply to the muscles of the leg, in case of occlusion of the femoral artery proximal to the profunda femoris artery [16]

On the other hand, it is noteworthy that most of the muscles affected are innervated from lower lumbar and upper sacral roots and in particular from the L4, L5, and S1, where the pathology was located. Therefore, the etiology of the neurophysiologic presentation of the pa-

Table 1. Innervation of the muscles affected in the present case

\begin{tabular}{|c|c|c|c|c|}
\hline Muscle & Innervation & Branch of & Branch of & Root \\
\hline Abductor hallucis & Medial plantar $\mathrm{n}$. & Tibial n. & Sciatic n. & $\mathrm{S} 1, \mathrm{~S} 2$ \\
\hline Tibialis anterior & Deep peroneal n. & Common peroneal n. & & L4, L5 \\
\hline Gastrocnemius & Tibial n. & Sciatic $n$. & & S1, S2 \\
\hline Quadriceps & Femoral n. & & & L3, L4 \\
\hline Extensor digitorum longus & Deep peroneal $n$. & Common peroneal $\mathrm{n}$. & & L4, L5 \\
\hline
\end{tabular}


tient should be sought at the level of the spinal cord/ spinal nerves and could most likely be attributed to a reduced crosssectional area of the vertebral canal or intervertebral foramen. Previous studies in healthy adults showed that flexion of the lumbar spine does not significantly affect the size of the disc, or the diameter of canal, however it does lead to an overall increase in the area of the canal, resulting from an increase in the subarticular sagittal diameters bilaterally [17]. At the same time, an increase in the diameter and area of the dural sac is observed compared with the neutral position. In addition, it has been demonstrated that the lumbar intervertebral foramina have an increased crosssectional area in the flexed position [18]. Hence, having excluded other potential causes of MEP attenuation reported in the literature, it could be hypothesized that in the present case their attenuation was possibly caused by the direct compression of the dural sac by the existing pathology, as its cross-sectional area increased relatively to the unaffected diameter of the spinal canal. It is speculated that this bulging of the dural sac over the free disk fragment further exacerbated the pressure phenomena leading to the failure of autoregulatory mechanisms.

A similar case has been reported by Ammar et al. [19], in which the patient had also no neurological deficits postoperatively. The attenuation of MEP again was attributed to the biomechanical alterations of the spine due to the prone position.

\section{Conclusion}

One of the key elements for the success of a neurosurgical procedure is proper patient positioning. Still, even if all precautions are taken and potential pitfalls are accounted for, it is possible that neuronal insult might be caused provided the altered anatomy due to the pathology is present. Hence, the importance of timely intervention in cases of abnormal intra-operative neurophysiology is strongly recommended.

\section{Ethical Considerations Compliance with ethical}

guidelines

All ethical principles are considered in this article. The participant was informed about the purpose of the research and its implementation stages. He was also assured about the confidentiality of his information. Moreover, he was free to leave the study whenever He wished, and if desired, the research results would be available to him.
Funding

This research did not receive any specific grant from funding agencies in the public, commercial, or not-forprofit sectors.

\section{Authors contributions}

Conceptualization: Sotirios Apostolakis, Aikaterini Karagianni, Konstantinos Vlachos; Methodology: Sotirios Apostolakis, Eirini Chiotaki; Investigation: Sotirios Apostolakis, Aikaterini Karagianni, Konstantinos Vlachos; Writing-original draft: Sotirios Apostolakis; Writing-review \& editing: Sotirios Apostolakis, Eirini Chiotaki; Supervision: Konstantinos Vlachos.

\section{Conflict of interest}

The authors declare that there is no conflict of interest.

\section{References}

[1] Rozet I, Vavilala MS. Risks and benefits of patient positioning during neurosurgical care. Anesthesiology Clinics. 2007; 25(3):631-53. [DOI:10.1016/j.anclin.2007.05.009] [PMID] [PMCID]

[2] Lalkhen AG, Bhatia K. Perioperative peripheral nerve injuries Continuing Education in Anaesthesia Critical Care \& Pain. 2012 12(1):38-42. [DOI:10.1093/bjaceaccp/mkr048]

[3] Bagshaw ON, Jardine A. Cardiopulmonary complications during anaesthesia and surgery for severe thoracic lordoscoliosis. Anaesthesia. 1995; 50(10):890-2. [DOI:10.1111/j.1365-2044.1995 tb05857.x] [PMID]

[4] Edgcombe H, Carter K, Yarrow S. Anaesthesia in the prone position British Journal of Anaesthesia. 2008; 100(2):165-83. [DOI:10.1093/ bja/aem380] [PMID]

[5] Anderson RC, Emerson RG, Dowling KC, Feldstein NA. Attenuation of somatosensory evoked potentials during positioning in a patient undergoing suboccipital craniectomy for chiari i malformation with syringomyelia. Journal of Child Neurology. 2001; 16(12):936-9. [DO I:10.1177/088307380101601214] [PMID]

[6] Nathan N, Tabaraud F, Lacroix F, Mouliès D, Viviand X, Lansade A, et al. Influence of propofol concentrations on multipulse transcranial motor evoked potentials. British Journal of Anaesthesia. 2003; 91(4):493-7. [DOI:10.1093/bja/aeg211] [PMID]

[7] Pankowski R, Roclawski M, Dziegiel K, Ceynowa M, Mikulicz $\mathrm{M}$, Mazurek T, et al. Transient monoplegia as a result of unilateral femoral artery ischemia detected by multimodal intraoperative neuromonitoring in posterior scoliosis surgery: A case report. Medicine (Baltimore). 2016; 95(6):e2748. [DOI:10.1097/ MD.0000000000002748] [PMID] [PMCID]

[8] Akagi S, Yoshida Y, Kato I, Sasai K, Saito T, Imamura A, et al. External iliac artery occlusion in posterior spinal surgery. Spine. 1999; 24(8):823-5. [DOI:10.1097/00007632-199904150-00016] [PMID] 
[9] Tseng MD, Cappetto L, Majid K, Sternberg D, Settecerri JJ. Bilateral femoral artery ischemia detected by multimodality neuromonitoring during posterior scoliosis surgery: A case report. Spine. 2010; 35(16):E799-803. [DOI:10.1097/BRS.0b013e3181d5577d] [PMID]

[10] Vossler DG, Stonecipher T, Millen MD. Femoral artery ischemia during spinal scoliosis surgery detected by posterior tibial nerve somatosensory-evoked potential monitoring. Spine. 2000; 25(11):1457-9. [DOI:10.1097/00007632-200006010-00021] [PMID]

[11] Trammell TR, Friedlander JK, Reed DB. The effect of lower limb ischemia on somatosensory evoked potentials during spinal surgery. Report of two cases and review of the literature. Spine. 1993; 18(3):413-5. [DOI:10.1097/00007632-199303000-00023] [PMID]

[12] Khan NR, Muhlbauer MS. Differential diagnosis, treatment, and prognosis of peripheral nerve injuries associated with neurosurgical procedures. In: Arthur A, Foley K, Hamm CW, editors. Perioperative considerations and positioning for neurosurgical procedures - a clinical guide: Springer; 2018. pp. 251-64. [DOI:10.1007/978-3319-72679-3_20] [PMCID]

[13] Kim C, Blank J, McClain BC. Transient paraparesis after general anesthesia in a patient in the prone position. Anesthesiology. 1994; 81(3):775-7. [DOI:10.1097/00000542-199409000-00033] [PMID]

[14] Ofiram E, Lonstein JE, Skinner S, Perra JH. The disappearing evoked potentials: A special problem of positioning patients with skeletal dysplasia: case report. Spine. 2006; 31(14):E464-70. [DOI:10.1097/01.brs.0000222122.37415.4d] [PMID]

[15] Li XJ, Lenke LG, Thuet E, Tan LA, Tuchman A. Prone Position-Induced Quadriceps transcranial motor evoked potentials signal lossa case report. Spine Deformity. 2018; 6(5):627-30. [DOI:10.1016/j. jspd.2018.02.008] [PMID]

[16] Schuenke M, Schulte E, Schumacher U. The lower limb - Neurovascular Systems: Topographical anatomy. In: Ross LM, Lamperti $E D$, editors. Thieme atlas of anatomy - general anatomy and musculoskeletal system. Stuttgart: Thieme; 2010. pp. 484-509. https:// books.google.com/

[17] Chung SS, Lee CS, Kim SH, Chung MW, Ahn JM. Effect of low back posture on the morphology of the spinal canal. Skeletal Radiology. 2000; 29(4):217-23. [DOI:10.1007/s002560050596] [PMID]

[18] Inufusa A, An HS, Lim TH, Hasegawa T, Haughton VM, Nowicki $\mathrm{BH}$. Anatomic changes of the spinal canal and intervertebral foramen associated with flexion-extension movement. Spine. 1996; 21(21):2412-20. [DOI:10.1097/00007632-199611010-00002] [PMID]

[19] Ammar A, Zarnegar R, Yassari R, Kinon M. Large central lumbar disc herniation causing acute cauda equina syndrome with loss of evoked potentials during prone positioning for surgery Surgical Neurology International. 2018; 9:66. [DOI:10.4103/sni. sni_482_17] [PMID] [PMCID] 